\title{
Unicam Activity Framework (UAF)
}

\author{
Gagliardi R., Mauri M., Polzonetti A.* \\ Technological Department, University of Camerino, Italy
}

Copyright $\subset 2016$ by authors, all rights reserved. Authors agree that this article remains permanently open access under the terms of the Creative Commons Attribution License 4.0 International License

\begin{abstract}
This presentation illustrates the framework of processing performance of the faculty of the University of Camerino. The evaluation criteria are explained and the technological structure that allows automatic performance assessment available online anywhere and anytime. The designed framework is usually applied to the performance evaluation of staff working for Università di Camerino (ITALY). It has provided excellent results which were positively judged by those who underwent the assessment, who also found that it portrayed their work quite properly.
\end{abstract}

Keywords Cloud, Business Intelligence, Performance Evaluation

\section{Introduction}

Evaluation of academic quality, productivity and performance has a key role on academic improvement of any academic unit such as an academician, group of academicians, department, institution or country. Performance and productivity evaluation is always a hot issue for all kinds of organizations regardless of their business or operational area. Moreover, quality management systems have been developed and been applied in order to control, evaluate, assure and improve quality hence performance and productivity. As in other industries and businesses, similar management systems started to take place in the academic world.

The importance of academic quality evaluation is strongly related with goals and objectives of an academic unit. On the other hand, the evaluation helps being aware of current status in terms of productivity and performance. Thus, it can be inferred that the evaluation leads to being aware of current status and it supports setting meaningful short term and long-term goals. In other words, how meaningful goals set depends on how successful an evaluation conducted. Successful evaluation also depends on how successful indicators selected. In short, indicators, evaluation, awareness and goals are heavily correlated terms. [7]

An alternative approach to the provision of research and teaching would be a combination of institutional diversification together with individual specialization. In this scenario, although the university aims at providing both high-quality teaching and high-quality research, some faculty members specialize in one of them. In any case, institutional or individual specialization is rather exceptional and most universities worldwide seem to adopt the synergic approach, based on the complementarity between teaching and research, motivating their faculty members to perform a mix of the aforementioned tasks together with certain amount of administrative duties. Taking this observation as a positive indication on the perceived synergic relationship among different types of academic duties, the normative question is whether the actual mix of the corresponding inputs and outputs is the optimal, or, alternatively, whether it could be improved by a redefinition of the obligations and objectives set by governments and university authorities [1]

Most academic positions in universities require three types of activities, namely teaching courses, conducting research in their respective fields and performing administrative duties. The UAF framework has been implemented to evaluate how academics perform their activities. However, data quality (i.e., that is correctness, completeness and uniqueness) can also benefit from the adoption of the UAF framework. [2]

UNICAM has decided to implement a system to monitor and evaluate the activities of individual teachers/researchers in compliance with the European Charter for Researchers and the recommendations by the European Commission published on the Official Journal of the European Commission of 11th March, 2005 ${ }^{1}$, which specifies what follows: 'Employers and/or funders should introduce for all researchers, including senior researchers, evaluation/appraisal systems for assessing their professional performance on a regular basis and in a transparent manner by an independent (and, in the case of senior researchers, preferably international) committee. Such evaluation and appraisal procedures should take due account of their overall research creativity and research results, e.g. publications, patents, management of research, teaching/lecturing, supervision, mentoring, national or international

$1 \mathrm{http}: / /$ eur-lex.europa.eu/legal-content/EN/TXT/?uri=CELEX:32005H025 
collaboration, administrative duties, public awareness activities and mobility, and should be taken into consideration in the context of career progression. The evaluation of these activities requires collection of data. The university assigns to different entities (such as administrative staff and secretaries) different data to be maintained. This is done in order to enhance data quality that is correctness, completeness and uniqueness. Although uniqueness may be guaranteed (each data has single managing entity) completeness and correctness are not guaranteed. For instance, the entity can make mistakes while entering the data; the entity can miss some update of data (if needed). The UAF framework not only evaluates academics' activities but also improves data quality. More precisely, each entity (that is being monitored) is highly interested in checking his own data for inaccuracies that can negatively affect his performance index. In this regard the lesson we learned while using the UAF framework is that releasing data and assessment criteria to the monitored entities will greatly improve data quality.

When implementing UAF the information's completeness should be functional to the definition of any subsequent evaluations aimed at achieving different and specific objectives. Different weighting should be assigned to researchers' activities and the monitoring of individual researchers should also be integrated by other kinds of information, such as details of the facilities used, for example. The method also provides indications for the assessment of individual researchers aimed at measuring their overall commitment. The assessment considers all the activities mentioned in the monitoring, which are assigned different weighting.

Monitoring is carried out for each calendar year taking into account the previous three years (except for those cases where appointment/employment started during the three-year period in question). The evaluation allows for a comparison of the cost against the 'value' produced by the researcher in terms of commitment and results obtained. At the end of the assessment, each researcher will be given a rating, which defines their commitment and that may be translated into months. Such a rating will then be multiplied by the value of the unit of measure $(€ 5,833)^{2}$ and compared with the average value for the reference category the researcher belongs to ${ }^{3}$.

\section{Researcher Rating Card}

\subsection{Monitoring and Measurement of Research Activity}

2 The researcher's commitment is assigned a specific value using Euro/month as a unit of measure $(€ / \mathrm{M})$ and is calculated by dividing 70,000 (average cost in Euro of an Associate Professor) by 12 (€ 5,833).

3 The cost of one unit of staff has been quantified by the Italian Ministry for University and Research (MIUR) as follows: Annual cost of a Full Professor, Euro 100,000.00; Annual cost of an Associate Professor, Euro 70,000.00; Annual cost of a University Researcher/Temporary Researcher, Euro $50,000.00$.
This section includes both the research products and the relevant and verifiable activities related to the research. Currently, there are about 30 different items that are taken into consideration and that are going to be evaluated.

According to the type of item, some reference values come into play and, possibly, some corrective parameters too.

As regards scientific articles published in journals, within bibliometric sectors, a reference value is based on an estimate of the time devoted to their production by a researcher. It was estimated that a researcher may devote 3/10 of his/her working time to such an activity.

After obtaining (source ANVUR ${ }^{4}$ ) the number of articles published over a 10-year period by a researcher working within a given Discipline Scientific Sector (such a number being defined as ' $\mathrm{m}$ '), taking into account that 3 out of the 10 years in question were devoted to scientific production and that 1 point is equivalent to 1 month of work, each article was given a $36 / \mathrm{m}$ score. Such a score implicitly includes the evaluation of author numerosity too.

A corrective factor for such a score is the positioning of the journal in question within the quartiles of the citation ranking (source Journal Citation Report - Web of Science).

Within non-biometric sectors, such as, for example, law, architecture, economics and social sciences, the reference value is similar to the one of biometric sectors. The only difference is the estimate of the time devoted by the researcher to scientific production.

As a corrective factor, the journal's ranking also affects other items, such as reviews, judgment/sentence remarks, as well as the role or function assigned, i.e. whether the person is an editor or a member of an editorial board.

As regards other types of publications, such as conference proceedings, encyclopedia items, patents, etc., the author numerosity factor comes into play too.

Peculiar items pertaining to some disciplines are also considered, such as the production of geological maps, projects, architectural compositions, and the organization of scientific events, expositions and exhibitions.

In this section, even the researcher's commitment concerning the presentation of research projects at various levels (i.e. European, national or regional ones) is rewarded too, even if no funding is linked to such projects.

In this last case, the source of data is the database of the central administration office that is devoted to the accountancy and administrative management of research projects.

\subsection{Monitoring and Assessment of Educational Activities}

The following activities are monitored and evaluated:

- The production of study books used for teaching university students;

4 Agenzia Nazionale di Valutazione del Sistema Universitario e della Ricerca. http://www.anvur.org 
- The production of written texts or chapters for university study books;

- Thesis supervision;

- The supervision of PhD students;

- The hours devoted to institutional or post-graduate lecturing on site, at any associated premises, or within other courses held abroad and managed by the university.

As regards the hours of lecturing, the score for every hour is 0.025 points $^{5}$ (see note) and 5 corrective factors have also been envisaged, which are respectively linked to:

1. The evaluation by students expressed in questionnaires concerning the teacher's performance in terms of subject knowledge, presentation quality, availability, punctuality;

2. Lecturing activity on site or off site;

3. Number of students in the classroom;

4. Language used for lecturing;

5. Lecturing within e-learning courses.

The score given to thesis supervision is linked to the role played by the teacher as supervisor, advisor or tutor, and to the type of study course the student enrolled for, i.e. bachelor, master, 5-year degree.

\subsection{Monitoring and Assessment of Funding}

This refers to the funds managed by UNICAM. The relevant figures are to be equally split and distributed amongst the entire internal researcher involved in the funded research.

The evaluation concerns the quota of EU funding that the university obtains as overheads, under a 'PQ' code, other international funding, national funding obtained through competitive programs (e.g. PRIN and FIRB), research funding from government or private bodies. Different scores and overheads are assigned according to the type of funding.

Data are automatically captured from the accountancy and administration management system used for research projects.

\subsection{Monitoring and Assessment of Institutional Functions}

This concerns the work carried out by teachers on a voluntary basis that, most of the times, is not paid for. As in all previous cases, a score is assigned to every activity according to the time devoted to it (calculated in months within a given year).
The roles and functions considered include the following ones: rector, prorector, functions delegated by the rector, management of or accountability for the organization of scientific or teaching facilities. Besides the institutional functions, other roles are also taken into consideration, such as being a member of high-profile, national or international institutions or commissions.

The teacher should record his/her functions in the framework. For every kind of function there is a person responsible of the relevant data that is in charge of confirming (through a special interface within the framework) what the teacher in question stated about his/her functions.

Some non-cumulative mechanisms have also been set up.

\section{Framework Development}

The framework helps to develop comprehensive Business Intelligence solutions.

The platform is process-centric because it uses Workflow engine as central controller. Workflow engine uses process definitions to define the business intelligence process that it executes on the platform. The process may be easily customized and new processes can also be added. The platform includes components and reports that may be used to analyze the performance of such processes.

The platform is solution-oriented because its operations are specified in the definition process and in the operation documents that provide details of every single activity.

These processes and operations together define the solution for the business intelligence problem. The solution may be easily added to business procedures outside the platform. The solution definition may include a number of processes and operations.

The platform is composed of:

- Business Intelligence Framework - which provides logging, auditing, security, scheduling and ETL procedures, as well as web services, attribute repository and rules engine;

- Business Intelligence Components - including components for reporting, analysis, workflow, dashboards and data mining;

- Business Intelligence Workbench - which is a set of design and administration tools enabling analysts and developers to create reports, dashboards, analysis models and business intelligence processes;

- Desktop Inboxes - which deliver notices generated by processes and reports.

The following image shows the system's architecture. 


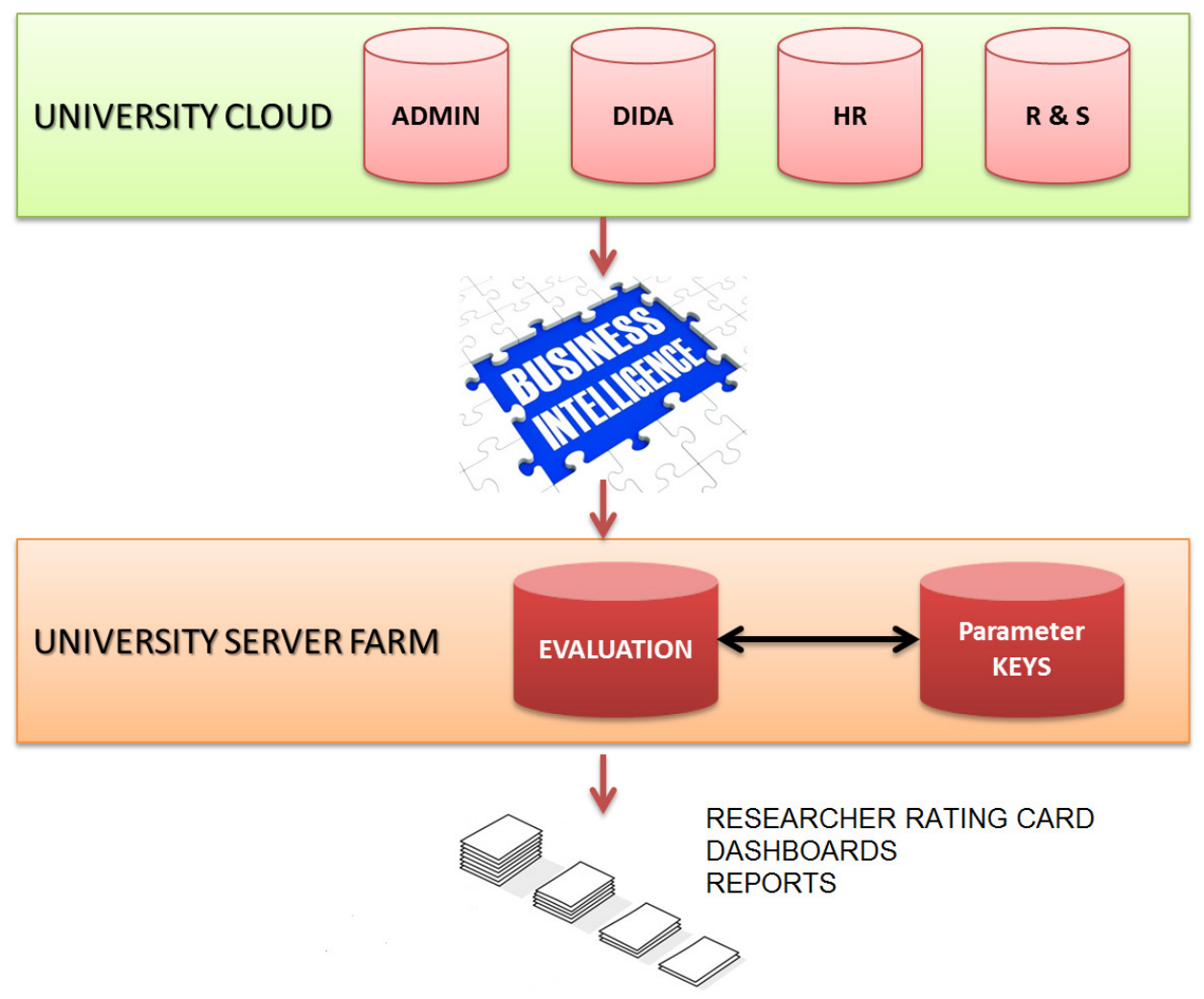

\subsection{Business Intelligence Framework}

The framework includes some special functions related to the flow of activities. The latter are governed by a workflow engine and are stored in the EVALUATION database that is used for data analysis, reporting purposes, and for the dashboards available to end users (mainly the university's management and teacher evaluators).

The main functions concern mining data from several sources that are shown in the relevant picture as 4 databases named: ADMIN, DIDA, HR, R\&S.

ADMIN is the database used to obtain information related to the projects managed by teachers that received funding, and to the proposals for projects without funding.

As already mentioned in Sections 3.1 and 3.3, both items are evaluated. The procedure for data mining also includes a processing phase for those projects whose funding amount is not known in advance. In such cases the amount is calculated for accountancy purposes by selecting and summing up the single payments received during the period considered.

DIDA represents the source of those data used to carry out the evaluations concerning the lecturing activity (Section 3.2). In this case, we are dealing with the most relevant amount of data, as they include the information concerning teachers' and students' careers, as well as the questionnaires completed by the latter.

In this case, the data mining process is highly selective and is focused on filtering information in order to extract only what is needed within the time interval in question. To do so, the system also uses some differential updating mechanisms.

R\&S contains the information related to the teachers' scientific publications that is used to extract the data needed to evaluate the research aspects specified in Section 3.1. This database will be fed by each teacher, every time that he/she uploads his/her publications through the IRIS ${ }^{6}$ application, which has recently replaced the old $\mathrm{U}_{-} \mathrm{GOV}^{7}$.

The IRIS database is quite important as it was used nationwide by $\mathrm{ANVUR}^{3}$ too for its VQR project, in order to evaluate the results of scientific research carried out by universities and research organizations. It is also going to be used to allocate (according to an award program) the ordinary funds that support the institutional work of the above players.

HR contains the information concerning the institutional work of every teacher, as well as other activities which are going to be considered for evaluation purposes that could not be obtained from the above-mentioned databases.

This database was created in parallel with the framework in question, together with the application that enables users to record their institutional work and that, at the same time, can also be accessed by those in charge of confirming the teachers' statements. The procedure includes a number of reports that are meant to support activity verification and checking.

Other information comes from heterogeneous sources. For example, the journal ranking (source Journal Citation Report - Web of Science) is available as an electronic data sheet.

6IRIS - Institutional Research Information System

http://www.cineca.it/it/content/IRIS

$7 \mathrm{U}-\mathrm{GOV}$ is an integrated IT system for the governance of universities and research organizations. http://www.cineca.it/it/content/il-sistema-u-gov 
The main process for the implementation of the workflow currently includes 40 activities. Each activity includes several individual operations. Following the necessary authentication, the process begins with the verification of the databases involved in the reading or writing activity. Once that phase has been successfully completed, the other operations are activated in a parallel or sequential mode. For any activities that require the connection to a database, the authentication phase is repeated. If an error occurs, the process stops and, anyway, the administrators are informed about the results of the whole process, as well as about every single activity carried out within the main process.

The job is scheduled to be carried out based on a given frequency that may vary according to the requirements envisaged, and that may also be increased in conjunction with evaluation processes.
To implement the workflow within the framework, Kettle software was used. Pentaho Data Integration (PDI, also called Kettle) is the component of Pentaho responsible for the Extract, Transform and Load (ETL) processes. Though ETL tools are most frequently used in data warehouses environments, PDI can also be used for other purposes and, in this particular instance, by using KETTLE's terminology representation the main workflow process was implemented through a job that includes the 40 or so changes that, in turn, are composed of an articulated series of processes.

The workflow system was installed on a LINUX server and Cron is used for the scheduling process, together with the operating system's shell. The picture below shows the first part of one of the main jobs. In the following picture the details of a change made within a job are shown.
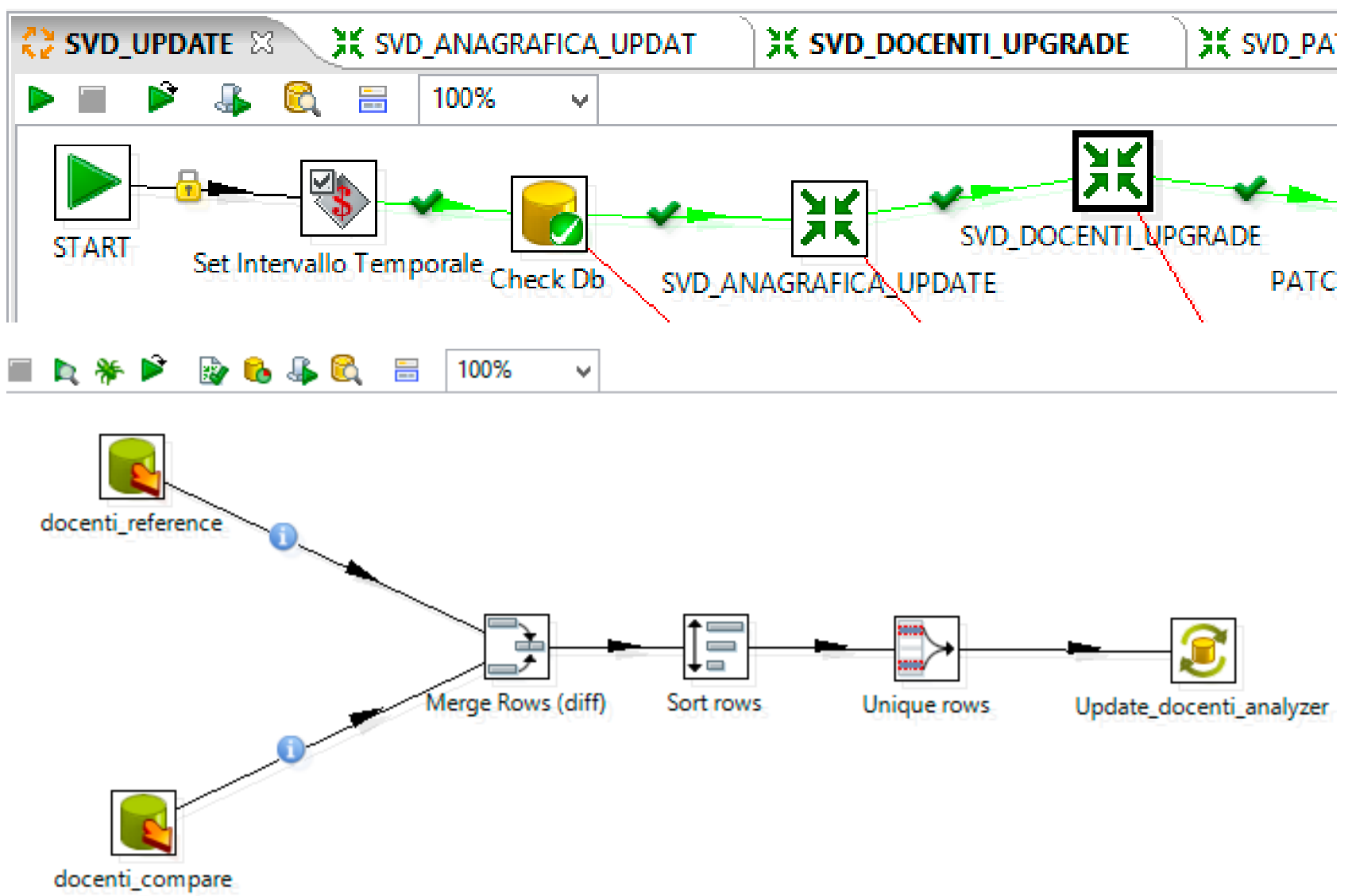


\subsection{Business Intelligence Components}

As regards the, Kettle functions are used to regulate the route throughout the various processes. A workflow can be graphically built by dragging the single items in the job and by linking them to each other as needed. The following picture shows a simplified part of the process concerning the uploading of journal ranking data on the EVALUATION database (see Section 3.1). The original data are provided every year by CRUI ${ }^{8}$ as electronic data sheets. Before being imported into the EVALUATION database, data need to be picked from the sheet and undergo some special processing. This activity is carried out through a process named Transformation 2. But before the process is activated some necessary conditions need to be verified through the process named Transformation 1. During the latter process, a given number of parameters are checked and, subsequently, the presence of the electronic data sheet within the file system is also verified (Process File Exist 1).

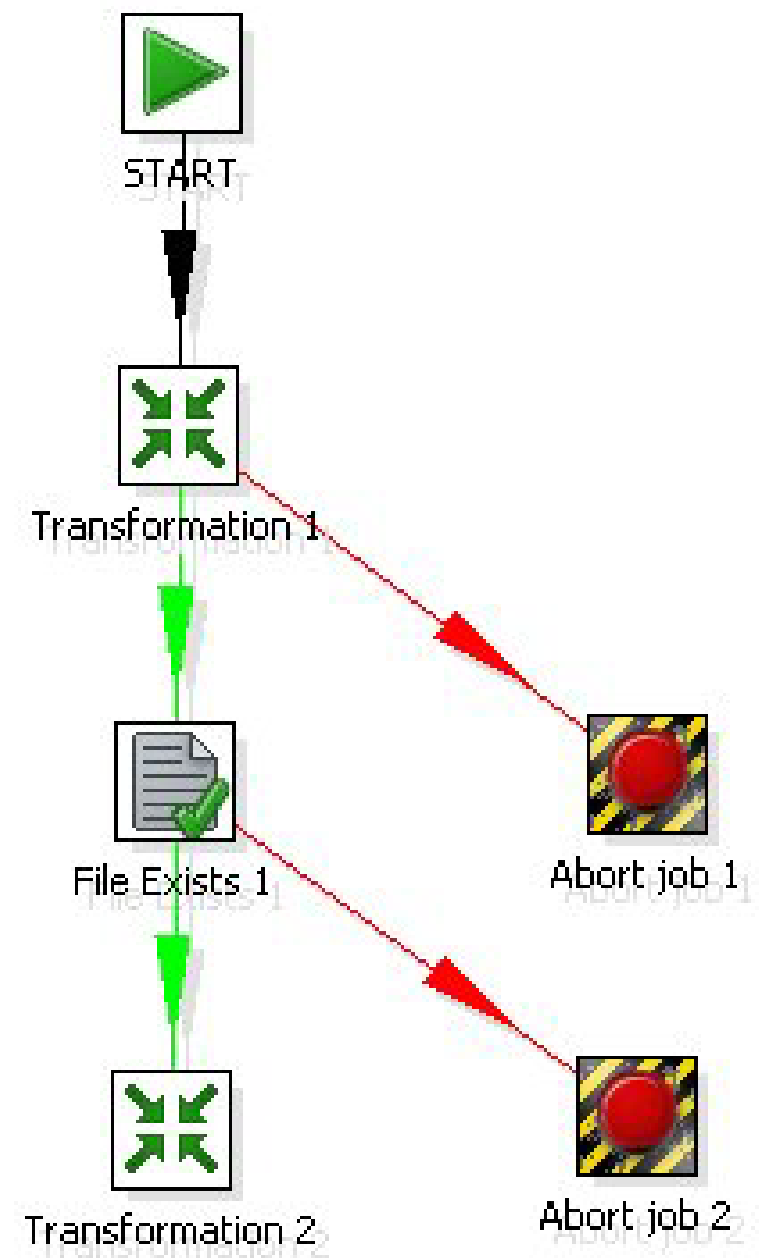

The available reports have been developed through the Community Edition of Pentaho Reporting that includes a suite of Open Source tools needed to create reports in PDF, Excel, HTML, Text, Rich-Text-File, XML and CSV formats. The reporting functions are carried out thanks to the JfreeReport ${ }^{9}$ engine that is owned and sponsored by Pentaho.

The main framework end user is the university management that prefers to have the results in a web page format, and as reports or dashboards.

The picture below shows an example of a web page containing the Research Rating Card as described in the previous sections.

$8 \mathrm{CRUI}$ is an association composed of Italian state and private universities that entered into an agreement with the Thomson Reuters publishing company to access the databases of Web of Science and Journal of Citation Reports (JCR).

9 JFreeReport is a free Java report library. It has the full on-screen print preview data obtained via Swing's TableModel interface based on report definitions, output to the screen, printer or various export formats, support for servlets. 


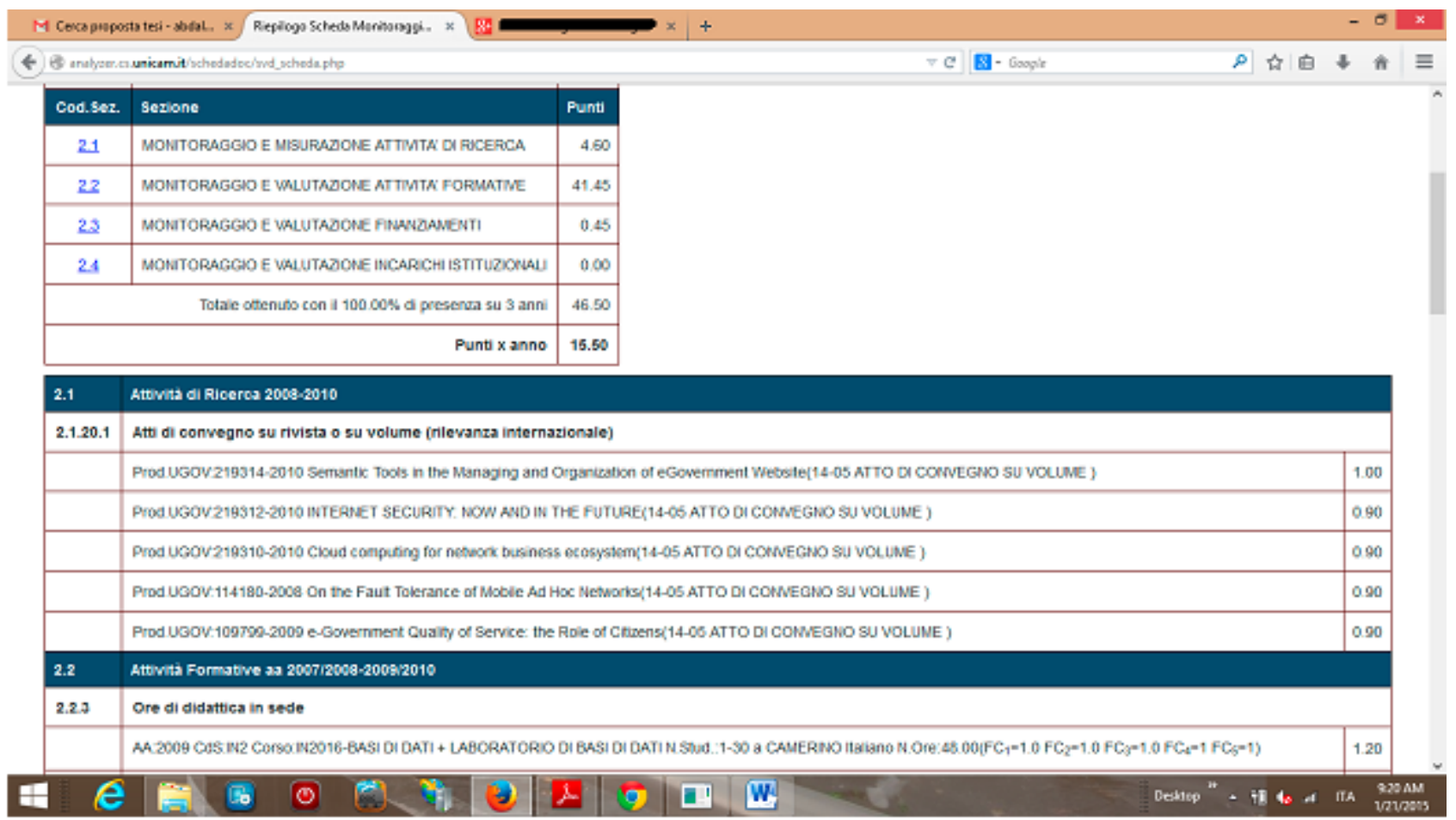

Within the EVALUATION database some data structures were developed according to the ROLAP model. Some tools that may be used to carry out multidimensional analyses are also available.

In particular, an interface developed through the SAIKU tool enables users to explore data sources by applying the familiar 'drag and drop' technique through a web page.

Finally, the university management can also use a series of dashboards that provide a concise view of the data, which are aggregated at various levels: by individual teacher, by role (researcher, senior fellow, full professor), by school, seen as a result of the aggregation, by individual facility, by faculty and by department.

The dashboard of a teacher is shown in the picture below as an example.

Users may select a given academic year and a particular teacher, and the relevant results will be displayed on the dashboard as:

1. A bar graph containing the following information:

a. The scores obtained by the teacher in the 4 sections displayed as a bar graph (green bars).

The scores can be compared with the lines showing the teachers' average score for each section.

b. The scores of the scientific area of the teacher (blue line)

c. The scores of the same role (red line)

d. The scores of the same Discipline Scientific Sector (yellow line)

e. The scores of the whole university (black line) 

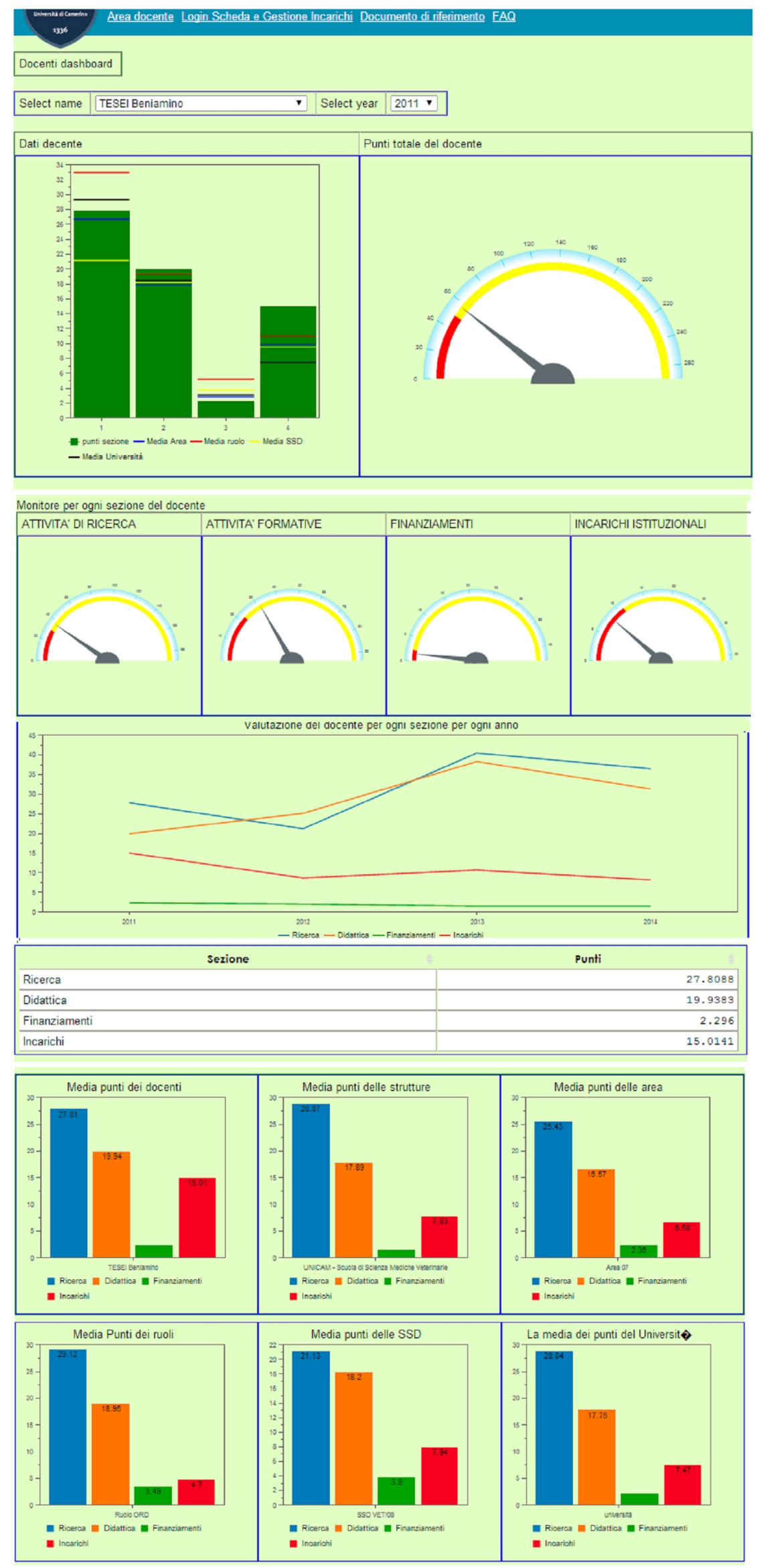


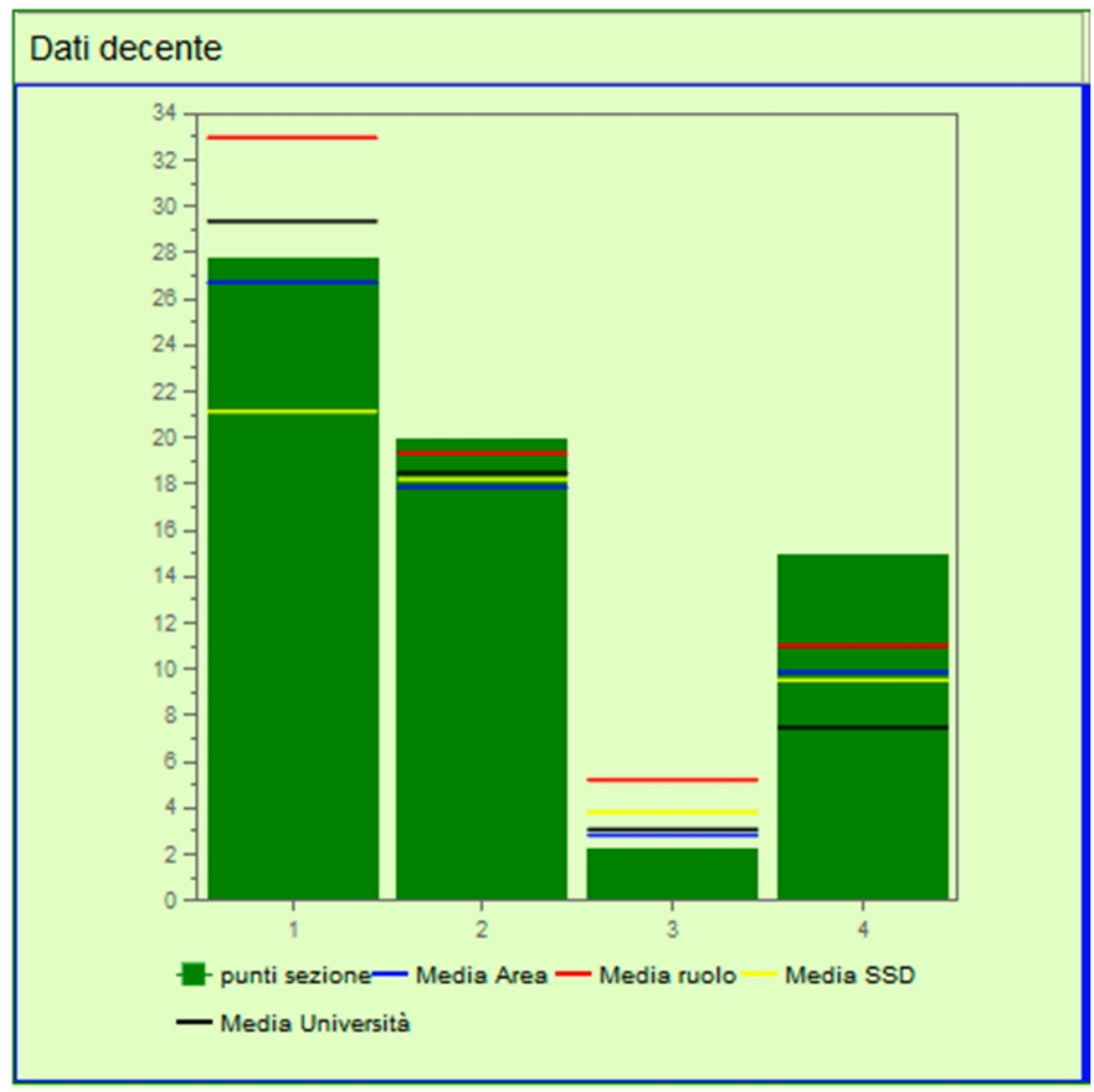

2. A Gauge Chart that compares the teacher's overall score (shown by the indicator with the minimum, medium and maximum overall score) calculated amongst all the teachers evaluated. Red and yellow identify respectively the scores that are above and below average.

\section{Punti totale del docente}

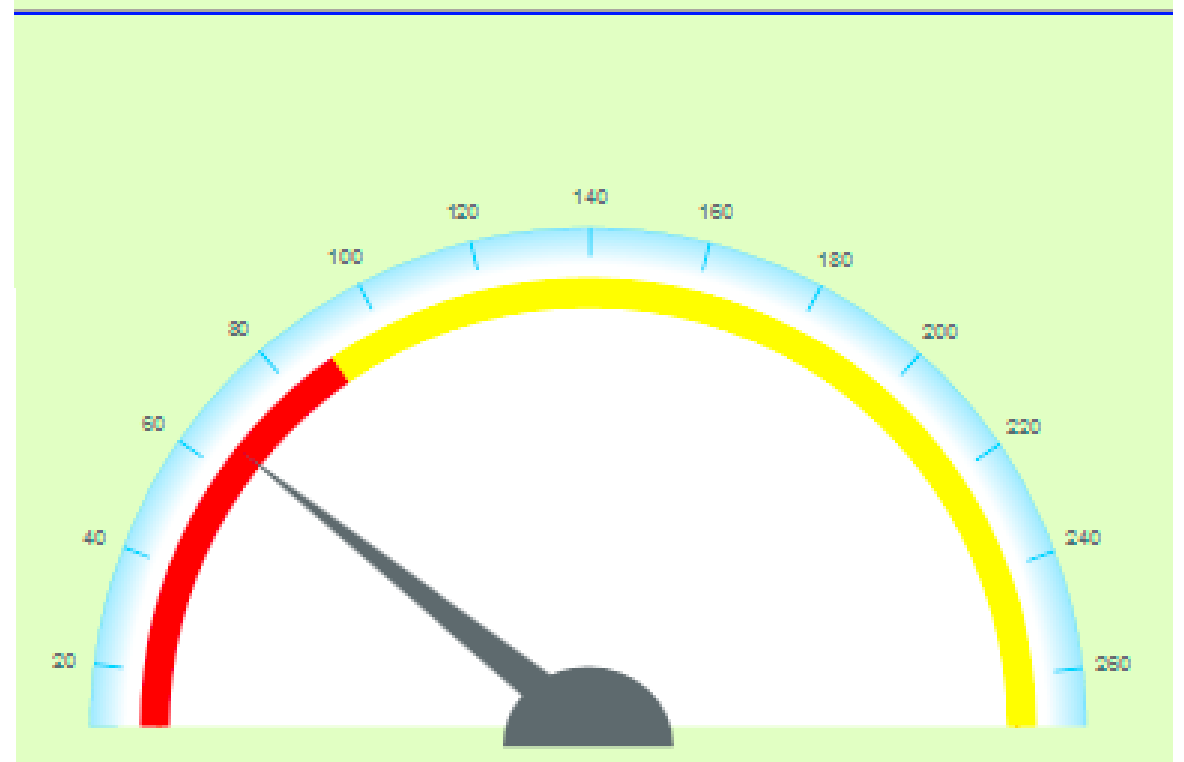

3. Four gauge charts show the scores obtained by the teacher in each one of the 4 sections by comparing them with minimum, medium and maximum scores obtained by all the teachers in the same sections. 


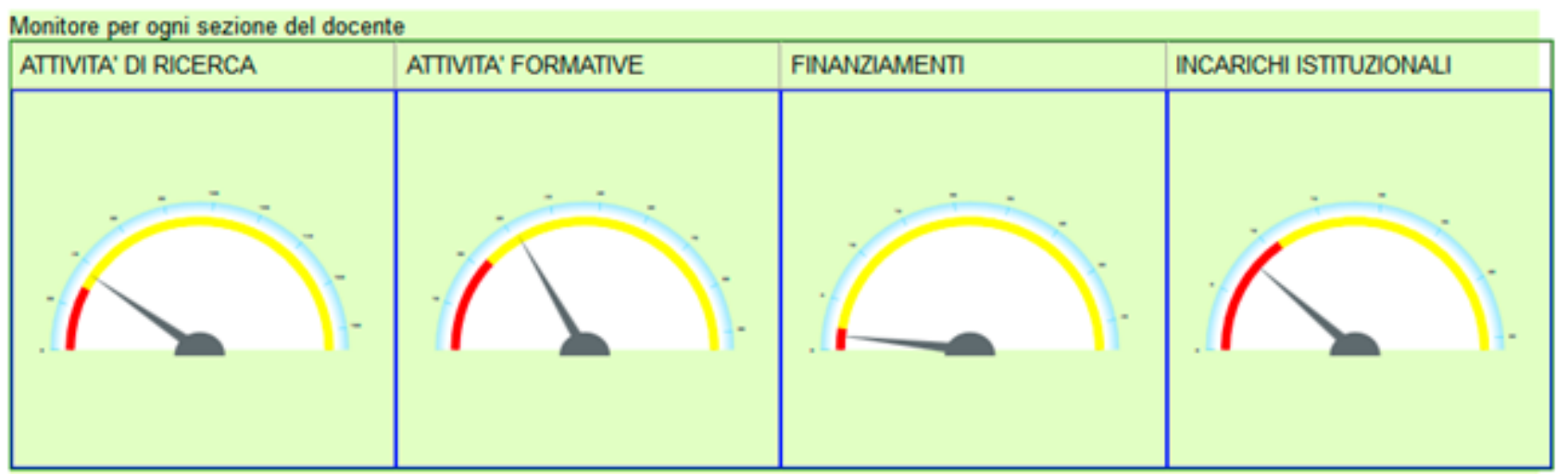

4. A Line Chart that shows the scores obtained in each section by the teacher in the selected year and, for comparison purposes, in the three previous years.

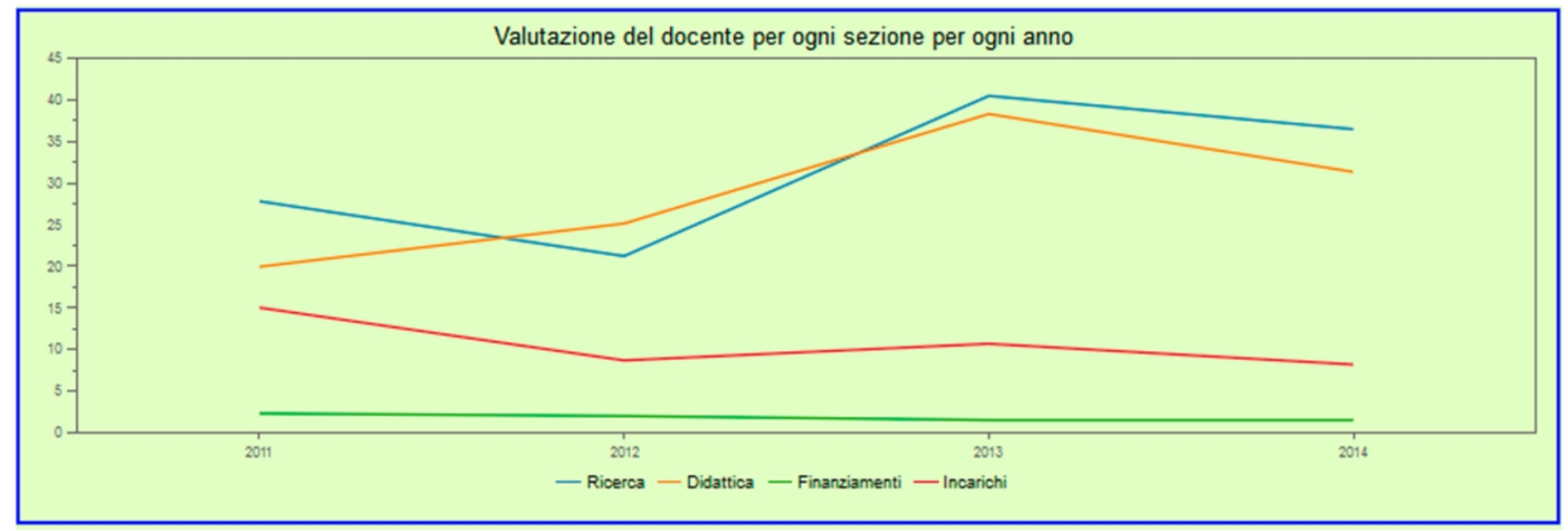

5. A table showing the scores obtained in the selected year.

\begin{tabular}{|l|r|}
\hline \multicolumn{1}{|c|}{ Sezione } & \multicolumn{1}{|c|}{ Punti } \\
\hline Ricerca & 36.4713 \\
\hline Didattica & 31.335 \\
\hline Finanziamenti & 1.4937 \\
\hline Incarichi & 8.1777 \\
\hline
\end{tabular}

6. A set of bar charts showing:

a. The scores obtained by the teacher in each section

The average scores of teachers working:

b. within the same school

c. within the same scientific area

d. in the same role

e. within the same Discipline Scientific Sector

f. within the whole university 


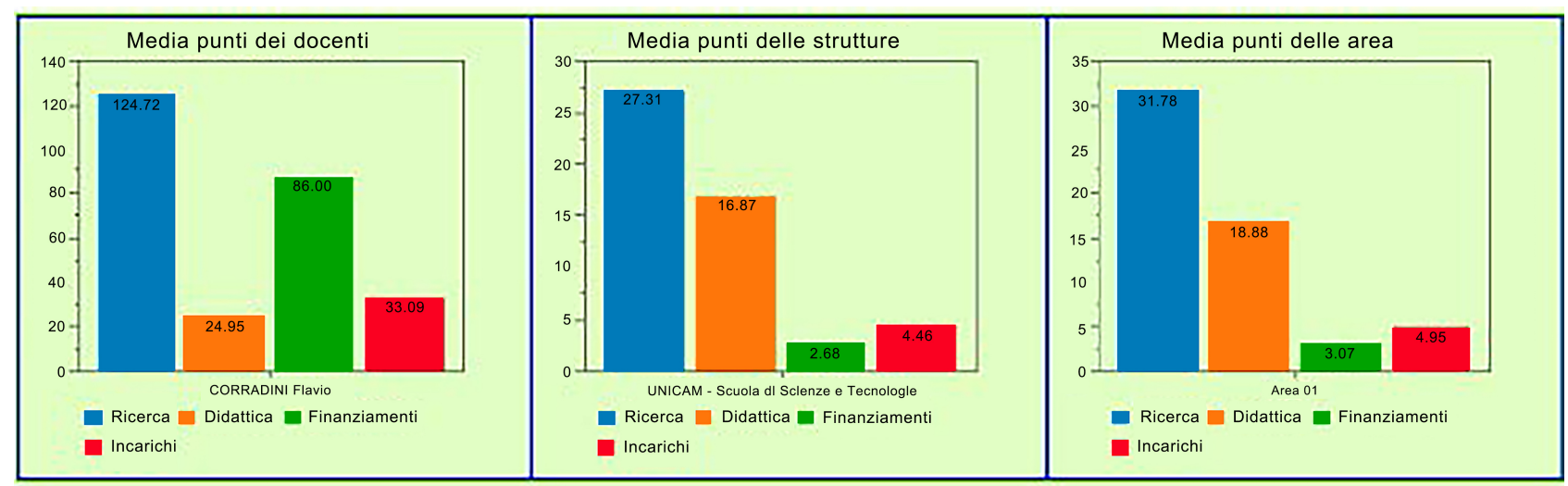

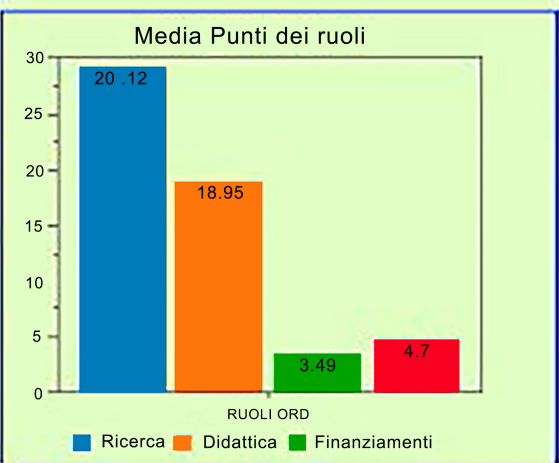

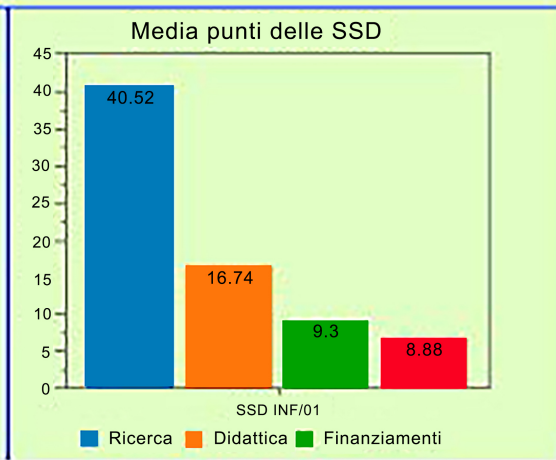

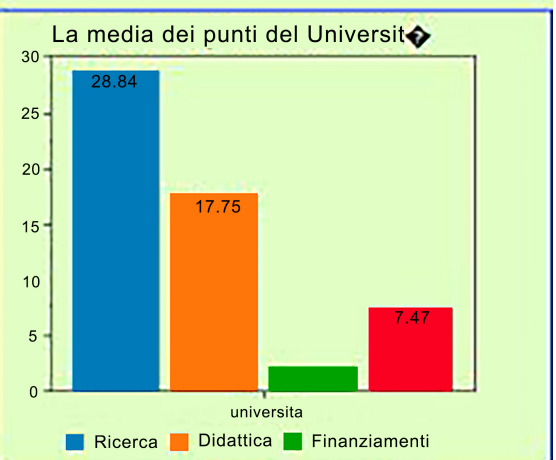

\subsection{Business Intelligence Workbench}

The set of tools available with the Pentaho Data Integration (PDI, also called Kettle) and the Pentaho BI Server Community Edition allowed us to provide the developers, and sometimes analysts too, with the tools needed to create whatever was required for the framework development.

Pentaho's BI Server or BA platform allows you to access business data in the form of dashboards, reports or OLAP cubes via a convenient web interface. Additionally it provides an interface to administer your BI setup and schedule processes.

In particular, using the Pentaho BI Server as a reference, we used the CDE Community Dashboard Editor and all the technology underneath it - namely CDF, CDA and CCC allows the development and deployment of Pentaho Advanced Dashboards. Community Dashboard Editor (CDE) was born to simplify the CTools Dashboards' creation, edition and rendering processes, and it's a very powerful and complete tool, combining front end with data sources and custom components in a seamless way.

Community Data Access (CDA) is the plugin designed for accessing data.

CCC stands for Community Chart Components, the CTools charting library.

As regards the multidimensional analysis function, in addition to the Saiku tool used for the analysis rendering, to build the OLAP cubes we used Mondrian, which is included in the suite that is an open source OLAP (online analytical processing) server, written in Java. It supports the MDX (multidimensional expressions) query language and the XML for Analysis and olap4j interface specifications. It reads from SQL and other data sources and aggregates data in a memory cache.

\subsection{Desktop Inboxes}

The Inbox Alerter is an executable that needs to be installed on the machines of the users that wish to take advantage of its functionality. The Inbox Alerter provides many ease-of-use features such as:

- Notification of new workflow tasks

- Notification of report delivery

- Management of off-line content

The Inbox Alerter uses an RSS feed provided by the Pentaho Server. Any RSS Reader that supports authenticated feeds can be used to receive notifications from the server. For off-line content management the Pentaho Inbox Alerter is required. The Pentaho Inbox Alerter will be provided based on the demand for client platform.

\section{Conclusions and Future Development}

Academic jobs require simultaneous performance of a variety of duties, including teaching, research and administration captured in a large number of alternative performance measures available on each one of them. Assuming that teaching is a university's major contribution to the society, we have addressed the question whether teaching quality is affected by other academic duties. 
The system that was implemented allows for the evaluation of many aspects of the work carried out by university researchers in relation to the many duties that globalization has brought about for the development of academic organizations.

Therefore, the relative value of university teachers is not determined just by their scientific value, but also by their ability to teach, to follow the study route of their students and to attract funding for the development of applied research. Last but not least, the management skills of each player within a university system have become a fundamental parameter for the overall evaluation of performance, especially because this kind of activity has become extremely important for university education, in every country.

Moreover, the literature we reviewed $[9,10,4,8]$ clearly demonstrates that:

- In general, the quality of university teaching is positively affected by published research. Specifically, research output improves teaching quality for small amounts of research, below the median performance in our sample, reaches a maximum and decays slowly for higher values of research output. That is, usual amounts of research are positive for teaching, while high research output may hinder teaching quality. In fact, two thirds of the professors could improve their teaching by doing more research.

- The quantity of teaching has a positive effect on teaching quality for relatively low teaching loads. The quality peaks around the average teaching load observed in our sample. In summary, moderate amounts of teaching, are beneficial for the quality, while higher teaching loads may decrease teaching quality. Since the average teaching load was 8 in the period under study, the observed arrangement does not seem to be far from the optimum.

- The quality of teaching is not affected by heavy administration duties. This may be because these appointments entail mandatory reductions in the workload to compensate for administrative work.

- The quality of teaching is negatively affected by light administrative duties. This may arise because many of these duties do not entail a compensating reduction in teaching loads.

- The quality of teaching is positively related to the participation in say, relevant committees, such as self-evaluation commissions, creation/reform of degree curricula, etc.

- The quality of teaching is positively associated to the elaboration of books or multimedia teaching materials with ISBN.

- Faculty members at the Education Dept. obtain better teaching results than the rest of the sample.

The developed framework may be added to other projects which are suggested in relevant literature $[3,5,6]$. However, some of its features make it quite different:
The framework includes all the work carried out by a university researcher;

- It is directly connected with the university's IT system (that, in Italy, is supplied by the Ministry of Education to $90 \%$ of Italian universities);

- Given its natural evolution, different evaluation weighting criteria for various activities may be potentially set up, through a Business Intelligence tool; - The performance dashboard, which enables the management to know how their university is going in real time.

As future work we are planning to quantify the enhancement in data quality.

\section{REFERENCES}

[1] García-Gallego, A., Georgantzís, N., Martín-Montaner, J., \& Pérez-Amaral, T. (2015). (How) Do research and administrative duties affect university professors' teaching?. Applied Economics, (ahead-of-print), 1-16.]

[2] Garwe, E. C. (2013). The effect of institutional leadership on quality of higher education provision. Research in Higher Education Journal, 22, 1.

[3] Goldsmith, L. T., Doerr, H. M., \& Lewis, C. C. (2014). Mathematics teachers' learning: A conceptual framework and synthesis of research. Journal of Mathematics Teacher Education, 17(1), 5-36.

[4] Hallinger, P., Heck, R. H., \& Murphy, J. (2014). Teacher evaluation and school improvement: An analysis of the evidence. Educational Assessment, Evaluation and Accountability, 26(1), 5-28.

[5] Hicks, D. (2012). Performance-based university research funding systems. Research Policy, 41(2), 251-261.

[6] Kane, T., Kerr, K., \& Pianta, R. (2014). Designing teacher evaluation systems: New guidance from the measures of effective teaching project. John Wiley \& Sons.

[7] Martin, B. (2012). Are universities and university research under threat? Towards an evolutionary model of university speciation. Cambridge Journal of Economics, 36(3), 543-565.]

[8] Martínez-Caro, E., Cegarra-Navarro, J. G., \& Cepeda-Carrión, G. (2015). An application of the performance-evaluation model for e-learning quality in higher education. Total Quality Management \& Business Excellence, 26(5-6), 632-647.

[9] Oral, M., Oukil, A., Malouin, J. L., \& Kettani, O. (2014). The appreciative democratic voice of DEA: A case of faculty academic performance evaluation. Socio-Economic Planning Sciences, 48(1), 20-28.

[10] Polikoff, M. S., \& Porter, A. C. (2014). Instructional alignment as a measure of teaching quality. Educational Evaluation and Policy Analysis, 0162373714531851. 